\title{
Eficácia da auriculoterapia na dor no ombro - uma revisão integrativa
}

\section{Eficácia da auriculoterapia na dor no ombro - uma revisão integrativa}

\author{
Renata Ulrich Finkler ${ }^{1}$, Márcio San Martim² \\ 1 - Universidade Federal de Ciências da Saúde de Porto Alegre - UFCSPA, Porto Alegre, RS, \\ Brasil. Núcleo de Atenção à Saúde UNIMED, Jundiaí, SP, Brasil. \\ 2 - Academia Brasileira de Arte e Ciência Oriental - ABACO, Porto Alegre, RS, Brasil. Institu- \\ to de Cardiologia, Porto Alegre, RS, Brasil.
}

renatafinkler@hotmail.com

Palavras-chave:
Auriculoterapia; Dor;

Ombro.

\section{RESUMO}

Objetivo: realizar uma revisão de estudos que mostrem os tratamentos de auriculoterapia para dor no ombro e protocolos relatados em livros. Método: foi utilizada a Biblioteca Virtual em Saúde acessando as bases de dados LILACS e SCIELO, com os descritores "auriculoterapia" e "ombro", onde foram encontrados 5 estudos, publicados entre 2001 e 2015 e referências bibliográficas. Os critérios de inclusão foram estudos originais que abordassem sobre auriculoterapia e dor no ombro e livros que abordassem protocolos para dor no ombro. Os critérios de exclusão foram estudos de revisão de literatura. Após análise foi excluído 1 estudo, sendo utilizados 4 estudos, devido 1 estudo ser revisão de literatura. Também foram incluídos 5 livros, por apresentarem protocolos para dor no ombro. Resultados: na pesquisa realizada, os estudos demonstraram resultados positivos em relação à auriculoterapia para o ombro. Em um dos estudos verificou-se que a auriculoterapia utilizada juntamente com tuiná tem melhor eficácia. em outro estudo, viu-se melhores resultados com acupuntura. nos outros 2 estudos, que foi com pacientes com síndrome do ombro doloroso e LER/DORT, foi utilizada apenas auriculoterapia, com bons resultados para dor. Considerações finais: essa revisão nos faz refletir sobre a importância de mais publicações para avaliar a eficácia da auriculoterapia para essa patologia. A eficácia da terapia poderia diminuir uso de medicamentos e efeitos colaterais provenientes dos mesmos e gastos para o governo, já que é uma terapia de baixo custo. Também foram apresentados livros com protocolos para tratamento de patologias do ombro, o que pode auxiliar os profissionais nos tratamentos dos pacientes. Verificado que há diversos protocolos disponíveis diferentes, tanto nos livros quanto nos estudos avaliados.

\section{ABSTRACT}

Objective: to perform a literature review of studies about auriculotherapy treatments for shoulder pain and protocols reported in books. Method: the Virtual Health Library was used to access the LILACS and SCIELO databases, with the descriptors "auriculotherapy" and "shoulder". Five studies were found between 2001 and 2015 including bibliographical references. Inclusion criteria were original studies about auriculotherapy and shoulder pain and books that include protocols for shoulder pain. Exclusion criteria were literature review studies. After analysis, one study was excluded, and four studies were used. Additionally, five books were included. Results: the studies demonstrated positive results in relation to shoulder auricular therapy. One study verified that auriculotherapy together with tuina massage was more effective. Another study found better results when auriculotherapy was associated with acupuncture. The other two studies, in which patients with shoulder impingement syndrome and LER/DORT were submitted only to auriculotherapy, presented good results for pain. Final considerations: the present review points to the importance of more publications to assess the effectiveness of auriculotherapy for this pathology. The effectiveness of this therapy can reduce the use of drugs and their side effects and government spending, since it is a low-cost therapy. The results

Keywords: also yielded books with protocols to treat shoulder disorders, which may help professionals in the Auriculotherapy; Pain; treatment of patients. Furthermore, there are several different protocols available, both in the books Shoulder. and in the studies evaluated. 


\section{INTRODUÇÃO}

O ombro é a articulação com maior mobilidade do corpo humano, sendo bastante complexa e instável. É necessário que haja sincronização entre todas as estruturas do ombro para uma biomecânica normal. Quando há alteração na estrutura e nas funções ocorrem inúmeras afecções. ${ }^{1}$

A dor é o principal motivo para procurar tratamento. Ter conhecimentos sobre a fisiopatologia da dor é dever de qualquer profissional de saúde. Muitos tratamentos mais complexos devem ser realizados por médico capacitado, mas a gestão geral da dor todos os profissionais de saúde devem estar aptos para tratar. ${ }^{2}$

A Política Nacional de Práticas Integrativas e Complementares (PNPIC) foi aprovada no Brasil em 2006. É denominada pela Organização Mundial da Saúde como medicina tradicional e complementar/ alternativa. As tecnologias que são utilizadas são eficazes e seguras, utilizando recursos terapêuticos e sistemas com complexidade. O cuidado, o auto-cuidado, a prevenção de doenças e a recuperação da saúde são estimulados com uma visão ampliada do processo saúde-doença. ${ }^{3}$

A Medicina Tradicional Chinesa (MTC) é uma medicina oriental que utiliza diversas práticas há milhares de anos. Muitas universidades e hospitais foram criados na China para prática dessa medicina no século XX, sendo oficializada por Mao Tze Tung. ${ }^{4}$

A fundamentação da MTC tem base na filosofia, onde o funcionamento do organismo humano, o ambiente e os ciclos da natureza estão interligados. O ser humano é visto como um todo, tendo uma abordagem humanista. É realizada a prevenção das doenças e o tratamento destas. ${ }^{4}$

Nesse estudo falaremos da auriculoterapia, mas na MTC existem mais 7 métodos diferentes, sendo eles: fitoterapia chinesa, moxabustão, dietoterapia (terapia alimentar chinesa), acupuntura, tuiná (massagem e osteopatia chinesa), ventosaterapia, práticas físicas (como Chi Kung, Tai Chi Chuan e algumas artes marciais) ${ }^{4}$

A auriculoterapia é um tratamento eficaz, na medida que é rápido, simples, de fácil aprendizagem, é prático e econômico e não apresenta efeitos colaterais. É utilizada para diversas finalidades como analgésica, hipotensora, antiestresse, sedação, estimulante, antipruriginosa, antifebril, entre outros. ${ }^{5,6}$

A Organização Mundial da Saúde (OMS) reconhece a auriculoterapia como um método auxiliar nos tratamentos terapêuticos e potencializadora de efeitos desejados. Os efeitos podem ser rápidos para diversos tratamentos e em outros podem precisar de mais sessões. $^{7}$

Ao longo do tempo, documentos foram encontrados falando de tratamentos semelhantes ao de auriculoterapia. Hipócrates tratava impotência com sangrias nas orelhas. Os egípcios utilizavam pontos da orelha para acalmar diversas dores. Em 1637, o médico Zacutus Lusitanus escreveu sobre cauterização auricular para tratamento de ciático. O professor Ignaz Colla, em 1810, descreveu sobre cauterizações auriculares para dores no ciático. O médico Lucciani, em 1850, falou também da cauterização da hélice para dor no ciático. Houve outras publicações no século. ${ }^{8}$

O pavilhão auricular apresenta grande inervação, ligando-se ao Sistema Nervoso Central. A terapia consiste em estimular pontos específicos, impulsionando o cérebro e fazendo com que sejam desencadeadas reações químicas e físicas. Essas reações reequilibram áreas e funções do corpo. $\mathrm{O}$ pavilhão auricular é um microssistema onde todo o organismo está representado. Os pontos estimulados regulam o sistema psíquico e orgânico. ${ }^{7,9}$

Todas alterações ou desequilíbrios do organismo são refletidos em regiões correspondentes no pavilhão auricular. É comumente visualizado na orelha manchas, dor e outros sintomas como conseqüência desses desequilíbrios, o que caracteriza o ponto. São utilizadas agulhas e sementes nos pontos até a cura completa ou até um alívio dos desequilíbrios. ${ }^{5}$

Cada ponto da orelha corresponde a uma parte do corpo, conforme um feto projetado no pavilhão auricular, em posição de nascimento, onde a cabeça ficaria no lóbulo da orelha e os pés na parte de cima. Fazendo essa analogia, os pontos estimulados correspondem aos órgãos do corpo. ${ }^{5}$

Por meio desta pesquisa foi identificada, nos trabalhos publicados, estudos que mostram a auriculoterapia como tratamento complementar à dor no ombro. Atualmente, está crescendo a utilização de práticas complementares no tratamento de diversas doenças. É importante saber como está sendo utilizada a auriculoterapia no tratamento da dor no ombro, visto que é um sintoma comum na população. Também estimulará os profissionais a procurarem a formação na terapia para que mais pacientes se beneficiem dessa prática.

A questão norteadora para esse estudo foi: Qual a eficácia da auriculoterapia no tratamento para dor no ombro e quais os tratamentos com auriculo- 
terapia são utilizados para tal patologia? A eficácia da terapia seria importante, na medida em que ela é relativamente de baixo custo, o que diminuiria gastos ao governo. Também diminuiria o consumo de medicamentos, auxiliando na saúde da população atendida. Esse estudo contribuirá para o conhecimento dos profissionais de saúde, pois mostrará alguns estudos realizados a respeito do tema em um único artigo.

\section{MÉTODO}

O estudo foi desenvolvido por meio da revisão integrativa da literatura através de artigos publicados entre 2001 e 2015, justifica-se por conter poucos estudos referentes ao tema na pesquisa realizada. Foram utilizados os descritores auriculoterapia e ombro no levantamento na Biblioteca Virtual em Saúde e foram encontrados estudos nas bases de dados SCIELO (Scientific Electronic Library Online) e LILACS (Literatura Latino-Americana em Ciências de Saúde). Os critérios de inclusão foram estudos originais que abordassem sobre auriculoterapia e dor no ombro. Foram excluídos estudos de revisão de literatura. Foram encontrados 5 (seis) estudos. Após análise dos artigos na íntegra, foi retirado 1 (um), sendo utilizados 4 (cinco) estudos, devido 1 estudo ser revisão de literatura. Dentre os estudos pesquisados, 2 são estudos internacionais. Também foram pesquisados livros (virtuais e não virtuais) que mostrassem protocolos de tratamentos para problemas relacionados ao ombro. Foram incluídos 5 livros, por apresentarem protocolos para dor no ombro.

Os estudos foram avaliados conforme critérios de exclusão e inclusão por meio de Teste de Relevância. Foram avaliados os 5 artigos na íntegra. No critério de inclusão foi respondido à questão: o estudo aborda o tema sobre auriculoterapia e dor no ombro? Também foi verificado se era artigo original ou revisão de literatura.

O financiamento do estudo foi realizado pelos autores. Não há conflitos de interesses. Não houve necessidade de submeter ao Comitê de Ética, por ser uma revisão integrativa.

\section{RESULTADOS E DISCUSSÃO}

A revisão realizada mostrará estudos realizados sobre a auriculoterapia e a dor no ombro e mos- trará alguns protocolos, registrados na literatura e utilizados para tratamento dessa patologia.

Foi realizado um estudo em Cuba, em Camagüey, onde os pacientes foram diagnosticados e diferenciados em 2 grupos de 30 pacientes cada. Um grupo utilizou somente a auriculoterapia e o outro grupo, auriculoterapia e tuiná. Os pontos auriculares utilizados foram shen men, rim, fígado e ombro. ${ }^{10}$

$\mathrm{O}$ estudo demonstrou que $65 \%$ dos pacientes evoluíram assintomáticos e 35\% melhoraram, tendo $100 \%$ eficácia do tratamento. A faixa etária que predominou foi de 45 a 54 anos (70\%). A intensidade da dor diminuiu em $98,3 \%$ e o ângulo de todos os movimentos do ombro aumentou em $100 \%$ dos pacientes. Os resultados indicam que os procedimentos são inócuos, úteis e eficazes, mas no tratamento combinado com auriculoterapia e tuiná o resultado foi alcançado após a quarta sessão, enquanto no tratamento apenas com auriculoterapia o sucesso foi alcançado na sétima sessão. Não houve reações adversas em ambos os esquemas utilizados. ${ }^{10}$

Já o estudo realizado em Umuarama/PR com pacientes com DORT e LER, com distúrbios tendinosos e musculares, principalmente em ombro, pescoço e membros superiores, foi realizado apenas com auriculoterapia. O objetivo do estudo era verificar a eficácia da mesma. Houve 12 participantes com sintomas dolorosos há mais de 6 meses, de ambos sexos, entre 30 e 50 anos. $^{11}$

O tratamento foi realizado com 10 sessões de auriculoterapia, sendo 2 vezes por semana. Era realizada sangria no ápice da orelha e os pontos utilizados foram shen men bilateral, rim bilateral, nervo simpático bilateral, fígado na orelha direita, baço-pâncreas na orelha direita e pontos correspondentes aos locais de dor, como ombro, articulação do ombro, cotovelo, punho, dedos e coluna cervical (unilateral ou bilateral). Os resultados obtidos concluíram que a auriculoterapia é eficaz no tratamento dessas doenças, pois diminui a dor e a conseqüente utilização de medicamentos e melhora da qualidade de vida das pessoas acometidas. $^{11}$

Igualmente o estudo realizado na Universidade Cruzeiro do Sul em São Paulo/SP foi somente com auriculoterapia. Teve por objetivo avaliar o tratamento com auriculoterapia para dor crônica, secundária à Sindrome do Ombro Doloroso, quanto ao efeito analgésico e terapêutico satisfatório e o tempo estimado de tratamento. Realizado um estudo de caso com 1 
participante que apresentava dor no ombro. A paciente tinha diagnóstico de artrose cervical com irradiação para o ombro, verificado através de tomografia computadorizada. $^{12}$

Foram realizadas 12 sessões de auriculoterapia. Foram utilizados os pontos shen men, rim, simpático, ombro, articulação do ombro e clavícula. $\mathrm{O}$ pavilhão auricular era alternado entre o direito e o esquerdo semanalmente. Concluiu-se que a auriculoterapia é uma técnica satisfatória, pois houve melhora relacionada à dor e à insônia causada pela dor. Evidenciou-se uma melhora de $34,3 \%$ na Síndrome do Ombro Doloroso da paciente estudada. ${ }^{12}$

No estudo realizado em Cuba, na Clínica Tula Aguilera, foi realizado uma comparação entre a efetividade da acupuntura e auriculoterapia no tratamento da bursite no ombro. Os pacientes foram divididos em 2 grupos, um tratado com acupuntura e outro com auriculoterapia. Cada grupo teve 90 pacientes, com idades entre 40 e 49 anos. ${ }^{13}$

O grupo tratado com acupuntura realizaram 10 sessões, sendo 1 sessão diariamente, descansaram 10 dias e iniciaram mais 10 sessões. Os pontos utilizados foram IG15, IG16, ID 13, VB34. A sessão durava 20 minutos e a cada 10 minutos os pontos eram estimulados. O grupo tratado com auriculoterapia realizava sessão a cada 7 dias por 4 semanas. Foram utilizados os seguintes pontos: ombro, clavícula, partes moles do ombro, tripla função, subcórtex e rim. ${ }^{13}$

Concluiu-se melhores resultados com acupuntura, sendo que $77 \%$ dos pacientes tiveram boa evolução, enquanto que com auriculoterapia $60 \%$ tiveram bons resultados. Quanto a resultado ruim foram 6 pacientes tratados com acupuntura e 24 pacientes tratados com auriculoterapia. Também com acupuntura os resultados obtidos foram mais rápidos, onde em 15 dias tiveram resposta positiva e com a auriculoterapia foi em 30 dias. $^{13}$

No livro Manual de Auriculoterapia foi apresentado um protocolo de pontos para problemas relacionados ao ombro. Os pontos mencionados foram: escápula, ombro, clavícula, pescoço, shen men, supra renal, occipital, córtex, rim, fígado, apêndice 2 , hélice 3 e amígdalas. ${ }^{14}$

O protocolo de pontos para o ombro no livro Noções práticas de auriculoterapia foi o seguinte: ombro como ponto principal, olho, trigêmeo, zero, membro superior, alergia, Darwin e occipital, como pontos complementares para perturbações de sensibilidade, e pontos pele e occipital para perturbações de motricidade. $^{8}$

Já no livro Auriculoterapia - Auriculomedicina na Doutrina Brasileira, os pontos mencionados para o tratamento de enfermidades no ombro foram: shen men, ombro, projeção do ombro no hélix, cervical, arcada dentária superior e inferior. Orienta que o ponto ombro seja colocado no pavilhão auditivo correspondente à dor, anterior e posterior. ${ }^{15}$

No livro Aurículo acupuntura, os pontos orientados foram shen men, ombro, articulação do ombro,

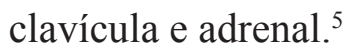

No livro Manual de auriculoterapia acupuntura auricular francesa e chinesa foram apresentados os pontos intestino delgado, intestino grosso e triplo aquecedor para alívio da dor, fígado e rim para lubrificar a articulação do ombro e nutrir os tendões e shen men, o principal ponto para alívio da dor. ${ }^{7}$

Nos 5 livros utilizados para esse estudo, 4 livros indicaram os pontos shen men e ombro. Outros pontos que foram citados em 2 livros diferentes foram: occipital, rim, clavícula, hélice e fígado.

\section{CONSIDERAÇÕES FINAIS}

Os 04 (quatro) estudos analisados descreveram sobre tratamentos realizados com auriculoterapia para problemas relacionados ao ombro, principalmente dor, mostrando os resultados e a eficácia encontrados com o uso da terapia. Na pesquisa realizada, os estudos demonstraram resultados positivos em relação à auriculoterapia para o ombro. Em um estudo foi mostrado que a acupuntura tem maior eficácia, mas mesmo assim, a auriculoterapia teve bons resultados.

Há uma grande deficiência de artigos publicados sobre o tema. Essa revisão mostra o quantitativo de estudos publicados, o que nos faz refletir sobre a importância de mais publicações para avaliar a eficácia da auriculoterapia para essa patologia. a eficácia dessa terapia poderia diminuir uso de medicamentos e efeitos colaterais provenientes dos mesmos e gastos para o governo, já que é uma terapia de baixo custo.

Também foram apresentados livros com protocolos para tratamento de patologias do ombro, o que pode auxiliar os profissionais nos tratamentos dos pacientes. Verificado que há diversos protocolos disponíveis diferentes, tanto nos livros quanto nos estudos avaliados. 


\section{REFERÊNCIAS}

1. Metzker CAB. Tratamento conservador na síndrome do impacto no ombro. Fisioter mov 2010;23(1):141-51.

2. Kopf A, Patel N. Guia para o Tratamento da Dor em Contextos de Poucos Recursos. Seattle: IASP Press ${ }^{\circledR}$ International Association for the Study of Pain, 2010.

3. Brasil. Portaria ${ }^{\circ}$ 971, de 3 de maio de 2006. Aprova a Política Nacional de Práticas Integrativas e Complementares (PNPIC) no Sistema Único de Saúde. Diário Oficial da União, Brasília, DF, 2006.

4. Blog Medicina Chinesa. Medicina Tradicional Chinesa. Disponível em: <http://www.medicinachinesapt.com/>. Acesso em: 01 de agosto de 2018.

5. Lee EW. Auriculo acupuntura. São Paulo: Editora Icone, 2010. 6. Haddad CRS. Terapia auricular. Rio de Janeiro: Editora IBTED, 2008.

7. Scavone AMP. Manual de auriculoterapia acupuntura auricular francesa e chinesa. Editora Kindle, 2016.

8. Nogier P. Noções práticas de auriculoterapia. São Paulo: Editora Andrei, 1998.

9. Brasil. Glossário temático: Práticas Integrativas e Complementares em Saúde. Secretaria de Atenção à Saúde, Secretaria-Executiva, Ministério da Saúde. Brasília, DF, 2018.

10. Vargas TJB. Eficacia de la auriculoterapia y combinación de auriculoterapia y tuina en la bursitis de hombro. Rev Cubana de Enfermer 2001;17(1):14-19.

11. Araujo APS, Zampar R, Pinto SME. Auriculoterapia no tratamento de indivíduos acometidos por distúrbios osteomusculares relacionados ao trabalho (DORT)/ lesões por esforços repetitivos (LER). Arq Ciênc Saúde Unipar 2006;10(1):35-42. doi: https:// doi.org/10.25110/arqsaude.v10i1.2006.140

12. Zanelatto AP. Avaliação da acupressão auricular na Síndrome do Ombro Doloroso: estudo de caso. Rev Bras Enferm 2013;66(5):695-701. doi: http://dx.doi.org/10.1590/S003471672013000500009

13. Cifuentes AD, Tejeda JMC, Iraola SV, Morales MDCC, Marrero DE. Efectividad de la acupuntura y la auriculoterapia en la bursitis de hombro de pacientes atendidos en el policlínico Tula Aguilera. Revista Electrónica Dr Zoilo E. Marinello Vidaurreta 2015;40(3):1-10.

14. Lipszyk M. Manual de Auriculoterapia. Buenos Aires: Editora Kier, 1989.

15. Mas WDD. Auriculoterapia - Auriculomedicina na Doutrina Brasileira. São Paulo: Editora Roca, 2005.

Como citar: FINKLER, Renata Ulrich; MARTIM, Márcio San. Eficácia da auriculoterapia na dor no ombro - uma revisão integrativa. Revista Interdisciplinar de Promoção da Saúde, Santa Cruz do Sul, v. 2, n. 1, jan. 2019. ISSN 2595-3664. Disponível em: $<$ https://online.unisc.br/seer/index.php/ripsunisc/article/view/13132>. Acesso em: 02 jan. 2019. doi: https://doi.org/10.17058/ rips.v2i1.13132 